\title{
Islands of runs of homozygosity indicate selection signatures in Ovis aries 6 (OAR6) of French dairy sheep
}

\author{
S. T. Rodríguez-Ramilo, ${ }^{1 *}$ A. Reverter, ${ }^{2}$ and A. Legarra ${ }^{1} \odot$
}

\section{Graphical Abstract}

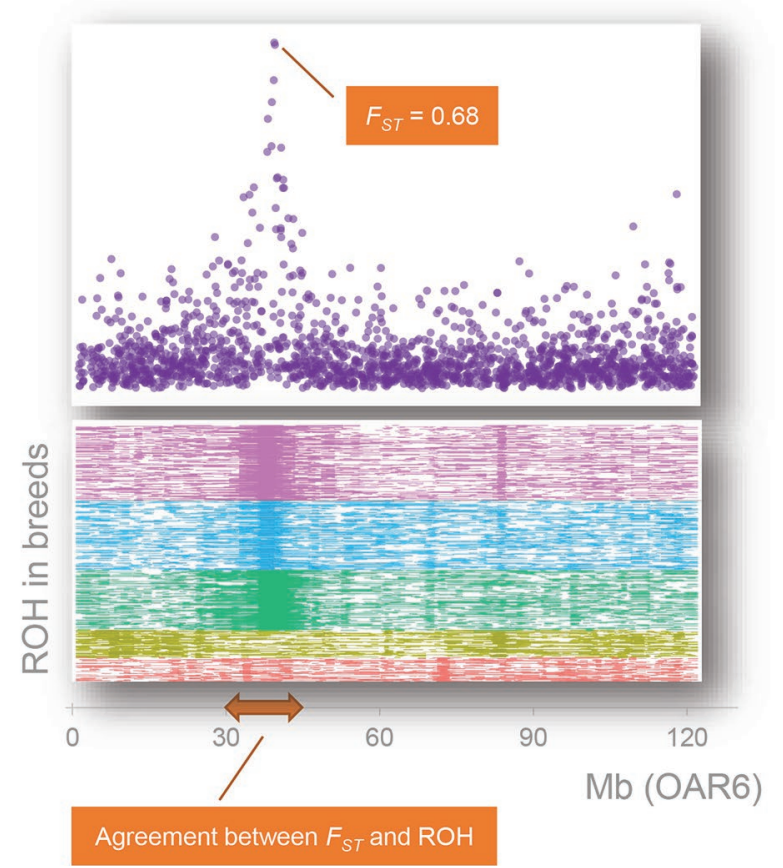

\section{Summary}

The occurrence of runs of homozygosity ( $\mathrm{ROH})$ is not randomly distributed across the genome, and ROH islands across many animals may be the result of selective pressure. The objective of this study was to demonstrate that the presence of $\mathrm{ROH}$ islands may be indicative of selection signatures in French dairy sheep breeds and subpopulations. One island of ROH was detected on OAR6 in the same genomic position across animals (between 30 and $40 \mathrm{Mb}$ ). Global Wright's differentiation coefficients $\left(F_{S T}\right)$ for 2 SNP (OAR6_41583796.1 and OAR6_41709987.1) within this ROH island were high (0.67-0.68). ROH and single SNP FST analyses agreed that selection signatures exist around both SNP that were located near the NCAPG and LCORL genes.

\section{Highlights}

- The presence of runs of homozygosity is not randomly distributed across the genome.

- Islands of runs of homozygosity may be the result of selection pressure.

- Concordance existed between islands of runs of homozygosity and selection signatures on OAR6.

- Candidate genes NCAPG and LCORL on OAR6 have agricultural and adaptive importance. 


\title{
Islands of runs of homozygosity indicate selection signatures in Ovis aries 6 (OAR6) of French dairy sheep
}

\author{
S. T. Rodríguez-Ramilo, ${ }^{1 *}$ A. Reverter, ${ }^{2}$ and A. Legarra ${ }^{1} \odot$
}

\begin{abstract}
Runs of homozygosity (ROH) are contiguous homozygous segments of the genome where the haplotypes inherited from each parent are identical. The occurrence of $\mathrm{ROH}$ is not randomly distributed across the genome, and $\mathrm{ROH}$ islands across many animals may be the result of selective pressure. The objective of this study was to demonstrate that the presence of ROH islands may be indicative of selection signatures in French dairy sheep breeds and subpopulations. The data set available included animals (artificial insemination males) from various breeds and subpopulations: Basco-Béarnaise breed (321 individuals), Manech Tête Noire breed (329 individuals), Manech Tête Rousse breed (1,906 individuals), Lacaune Confederation subpopulation (3,030 individuals), and Lacaune Ovitest subpopulation (3,114 individuals). Animals were genotyped with the Illumina OvineSNP50 BeadChip. After applying filtering criteria, the genomic data included 38,287 autosomal SNP distributed across 26 chromosomes and 8,700 individuals. One island of ROH was detected on OAR6 in the same genomic position across animals (between 30 and $40 \mathrm{Mb}$ ). Global Wright's differentiation coefficients for 2 SNP within this ROH island were high (0.67-0.68). The linkage disequilibrium between both SNP was also elevated (0.98). The divergence in allele frequencies in those SNP grouped Basco-Béarnaise, Manech Tête Noire, and Manech Tête Rousse breeds in one cluster and Lacaune Confederation and Lacaune Ovitest subpopulations in another cluster. The closest candidate genes are NCAPG and LCORL, which have been reported to be under positive selection and suggested to control weight and height in sheep. The preliminary identification of ROH suggests the presence of selection. However, for the identification of potential candidate genes, ROH detection should be combined with other approaches to improve mapping accuracy.
\end{abstract}

$S^{\prime}$ heep, one of the first domesticated species, have a manageable size and the ability to adapt to different climates and diets with poor nutrition (Zeder, 2008). From their domestication, sheep spread worldwide, and natural and artificial selection shaped a large variety of breeds with different morphology, coat colors, and specialized production in meat, milk, or wool. These selection mechanisms have left signatures in domestic animal genomes, such as decreased genetic diversity and long haplotypes (Rochus et al., 2018).

Different methods varying in the underlying assumptions have been proposed to detect selection signatures, such as the genomewide differentiation coefficient ( $\mathbf{F}_{\mathbf{S T}}$; Weir and Cockerham, 1984), extended haplotypes of homozygosity (Sabeti et al., 2002), and integrated haplotype homozygosity score (iHS; Voight et al., 2006). Genome-wide scans for detecting selection signatures have been successfully applied to domestic animals based on these approaches.

The availability of large numbers of SNP has made these markers appropriate to distinguish old (short runs) from new (long runs) identical by descent segments (Howrigan et al., 2011). Accordingly, SNP can be used for the detection of genomic regions where an increase in homozygosity has occurred. Although the presence of copy number variation or large distance between SNP can interfere with the detection of regions of homozygosity (Nandolo et al., 2018), the identification of runs of homozygosity (ROH) is a potential alternative to detect signatures of selection. The oc- currence of $\mathrm{ROH}$ is heterogeneous along the genome due to the stochastic nature of the recombination events. Consequently, the detection of $\mathrm{ROH}$ islands may be indicative of selective pressure. Runs of homozygosity can provide a better understanding about the population selection history, supporting the discovery of genes or genomic regions under selective pressure (Zhang et al., 2015).

Selection of French dairy sheep has been implemented for each local breed and subpopulation separately. There are 3 major regions for sheep milk production. The first is around the Roquefort (Aveyron) area, with the Lacaune breed, whose elite population (breeding flocks) consists of 2 subpopulations, Lacaune Confederation (LACCon) and Lacaune Ovitest (LACOvi), which split around 1975 and proceed in parallel selection schemes. In the second region, the Western Pyrenees mountains, there are 3 breeds: Manech Tête Rousse (MTR), Manech Tête Noire (MTN), and Basco-Béarnaise (BB). The third region is Corse, but Corsican sheep were not included in the present study. All of the breeds in this study (LACCon, LACOvi, MTR, MTN, and BB) have been selected for production (milk yield and composition) and functional (resistance to mastitis, udder morphology) traits with varying intensities. The objective of this study was to demonstrate that the presence of $\mathrm{ROH}$ islands may be indicative of selection signatures in French dairy sheep breeds and subpopulations.

Rams were genotyped with the OvineSNP50 BeadChip (Illumina Inc.). The number of genotyped animals was 321, 329, 1,906, 3,030, and 3,114 for BB, MTN, MTR, LACCon, and LACOvi,

\footnotetext{
${ }^{1}$ GenPhySE, Université de Toulouse, INRAE, ENVT, F-31326, Castanet Tolosan, France, ${ }^{2}$ CSIRO Agriculture \& Food, Brisbane, QLD 4067, Australia.

*Corresponding author: silvia.rodriguez-ramilo@inrae.fr. @ 2021, The Authors. Published by Elsevier Inc. and Fass Inc. on behalf of the American Dairy Science Association ${ }^{\oplus}$. This is an open access article under the CC BY license (http://creativecommons.org/licenses/by/4.0/). Received July 17, 2020. Accepted February 05, 2021.
} 
respectively. These are AI rams representing several years of birth (10-15) and overlapping generations, and they illustrate the genetic variability of each breed. The SNP quality control included the absence of parent-offspring Mendelian segregation incompatibilities $(<3 \%)$, SNP call rate $>97 \%$, and large deviations from Hardy-Weinberg equilibrium (SNP with $P<10^{-6}$ were discarded). The final data set included 8,700 genotyped individuals and 38,287 autosomal SNP. Markers were positioned using the sheep (Ovis aries) genome assembly 3.1 (OAR 3.1; https://www.ncbi.nlm.nih .gov/assembly/GCF_000298735.1/). See Legarra et al. (2014) for more details.

Plink software version 1.9 (Chang et al., 2015) was used to calculate the genetic differentiation coefficient $\mathrm{F}_{\mathrm{ST}}$ (Weir and Cockerham, 1984) between subpopulations and breeds with the option -fst. Significance level was evaluated using Lewontin and Krakauer (1973) statistical test (Bonhomme et al., 2010) and applying a Bonferroni correction using the total number of markers.

The rehh R package (Gautier and Vitalis, 2012) with the default values was used to calculate iHS, where an extreme score $(>2$ or $<-2$ ) provides a strong signal of selection (Voight et al., 2006), indicating the most extreme $5 \%$ of iHS values. The software detec-
tRUNS (Biscarini et al., 2018) was used to detect ROH with a sliding window-based method and the following criteria: a minimum length of $250 \mathrm{~kb}$, a minimum of 20 homozygous SNP, a maximum distance of $1 \mathrm{Mb}$ allowed between 2 consecutive homozygous SNP in a run, a minimum density of 1 SNP per $10 \mathrm{~kb}$, and no missing or heterozygous genotypes allowed.

The correlation between the number of times an SNP is included within an $\mathrm{ROH}$ and $\mathrm{F}_{\mathrm{ST}}$ (or iHS) was calculated for all SNP in each chromosome. The average genetic differentiation coefficient across breeds and subpopulations was $0.0576 \pm 0.0003$. The genome-wide distribution of global differentiation coefficient for each SNP revealed that the highest selection signal was detected on OAR6 (Figure 1a). The 2 highest ranked SNP (OAR6 41583796.1, $\mathrm{F}_{\mathrm{ST}}=0.675, P=4.03 \times 10^{-9}$; and OAR6 41709987.1, $\mathrm{F}_{\mathrm{ST}}=0.670$, $P=4.01 \times 10^{-9}$ ) were located at $37,423,374$ and $37,542,319 \mathrm{bp}$, respectively. Three other significant SNP were also detected on OAR6 near the 2 highest ranked SNP at 36,205,101, 36,972,847, and $37,318,895 \mathrm{bp}$ ). In addition, 6 isolated significant SNP were detected on OAR3 (2 SNP; 61,548,300 and 61,577,699 bp), OAR11 (1 SNP; 40,118,817 bp), OAR13 (2 SNP; 46,841,439 and $62,932,554 \mathrm{bp}$ ), and OAR16 (1 SNP; 33,219,172 bp). The $\mathrm{F}_{\mathrm{ST}}$
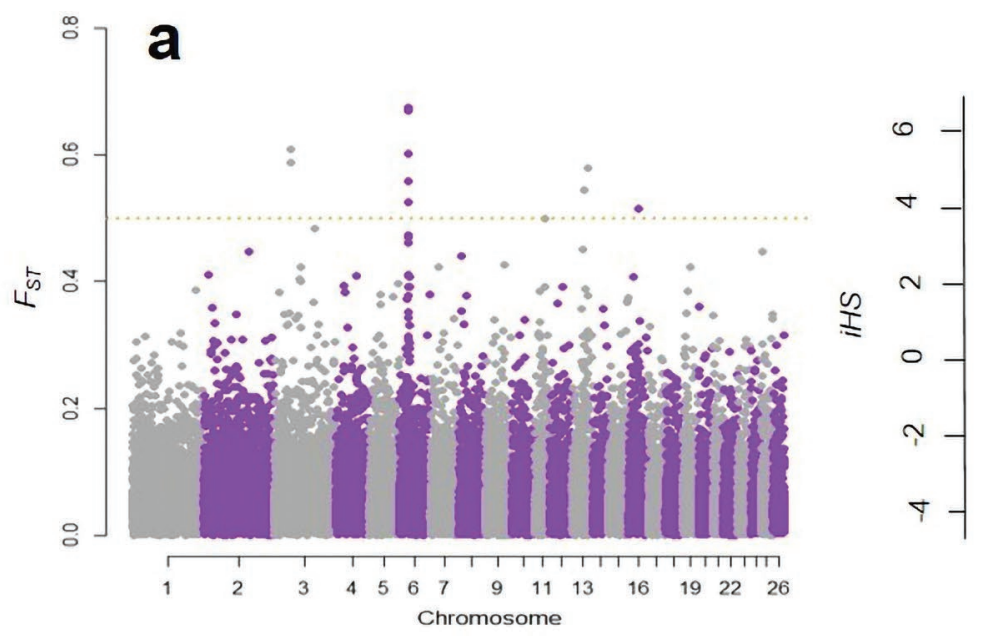

b
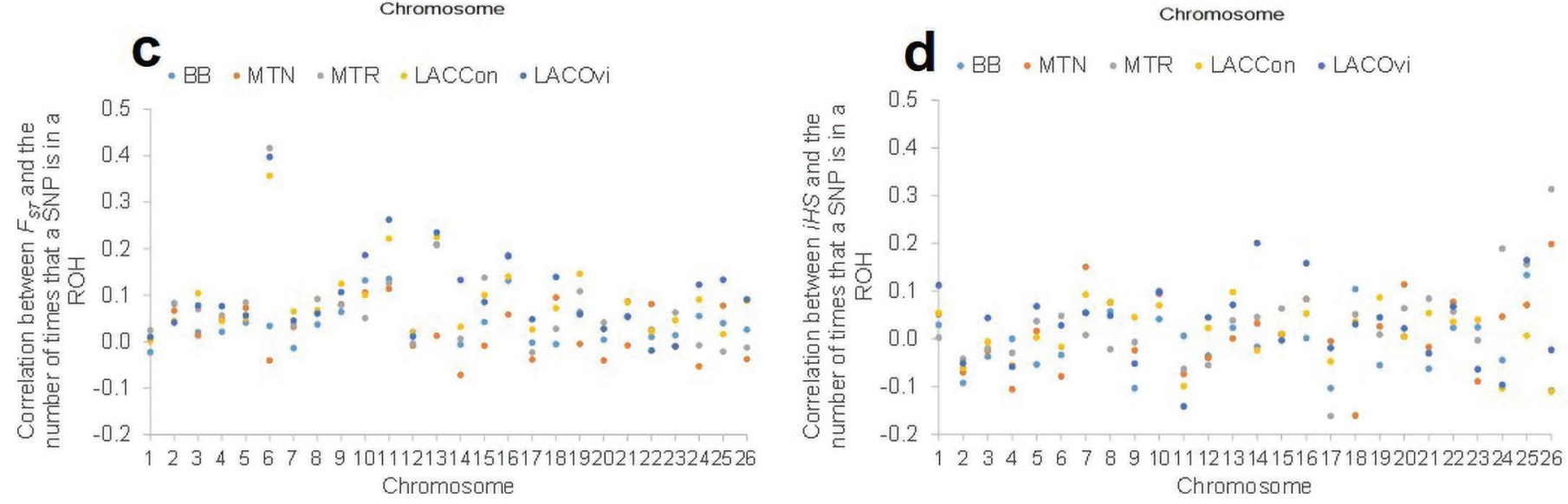

Figure 1. (a) Genome-wide differentiation coefficient $\left(F_{S T}\right)$ across chromosomes. Orange dashed line indicates the significance level after Bonferroni correction. (b) Integrated haplotype homozygosity score (iHS). Black dashed lines indicate the iHS threshold ( $>2$ or $<-2$ ). (c) Correlation between $\mathrm{F}_{\mathrm{ST}}$ and the number of times that an SNP is in an ROH. (d) Correlation between iHS and the number of times that a SNP is in an ROH. BB = Basco-Béarnaise; MTN = Manech Tête Noire; MTR = Manech Tête Rousse; LACCon = Lacaune Confederation; LACOvi = Lacaune Ovitest. 
Table 1. Differentiation coefficient between pairs of breeds and subpopulations for SNP OAR6_41583796.1 (above the diagonal) and OAR6_41709987.1 (below the diagonal)

\begin{tabular}{lccccc}
\hline Breed $^{1}$ & BB & MTN & MTR & LACCon & LACOvi \\
\hline BB & & 0.077 & 0.131 & 0.652 & 0.777 \\
MTN & 0.076 & & 0.403 & 0.481 & 0.639 \\
MTR & 0.129 & 0.397 & & 0.784 & 0.860 \\
LACCon & 0.649 & 0.479 & 0.782 & & 0.019 \\
LACOvi & 0.771 & 0.633 & 0.856 & 0.018 & \\
\hline
\end{tabular}

${ }^{1} \mathrm{BB}=$ Basco-Béarnaise; MTN = Manech Tête Noire; MTR = Manech Tête Rousse; LACCon = Lacaune Confederation; LACOvi = Lacaune Ovitest.

values for the remaining autosomes were not as high as the $\mathrm{F}_{\mathrm{ST}}$ values observed for the 2 highest ranked SNP on OAR6. Results obtained for the iHS derived statistic are shown in Figure 1b. A total of 1,735 significant SNP were detected in all chromosomes for the iHS statistic. Agreement between significant SNP for $\mathrm{F}_{\mathrm{ST}}$ and iHS was detected only for 1 SNP on OAR13 on 46,841,439 bp. A total of 95 significant SNP for iHS were observed in OAR6. At least 2 significant SNP for iHS on OAR6 (OAR6_39124095.1 and OAR6_42392875.1) were close $(35,063,421$ and 38,193,916 $\mathrm{bp}$, respectively) to the highest significant SNP for $\mathrm{F}_{\mathrm{ST}}$.

The highest correlation between $\mathrm{F}_{\mathrm{ST}}$ and the number of times that an SNP was in an ROH was observed in OAR6 for LACOvi and LACCon subpopulations and MTR breed ranging between 0.36 and 0.42 (Figure 1c). However, the highest correlation $(0.31)$ between iHS and the number of times that an SNP was in an ROH was observed in OAR26 for the MTR breed (Figure 1d). The correlation between iHS and the number of times that an SNP was in an ROH does not support the OAR6 signal observed in Figure 1c. This can be related to the fact that $\mathrm{F}_{\mathrm{ST}}$ and iHS have identified different significant SNP within OAR6.

The $\mathrm{F}_{\mathrm{ST}}$ values for SNP OAR6_41583796.1 and OAR6 41709987.1 between pairs of breeds and subpopulations are

\section{a}

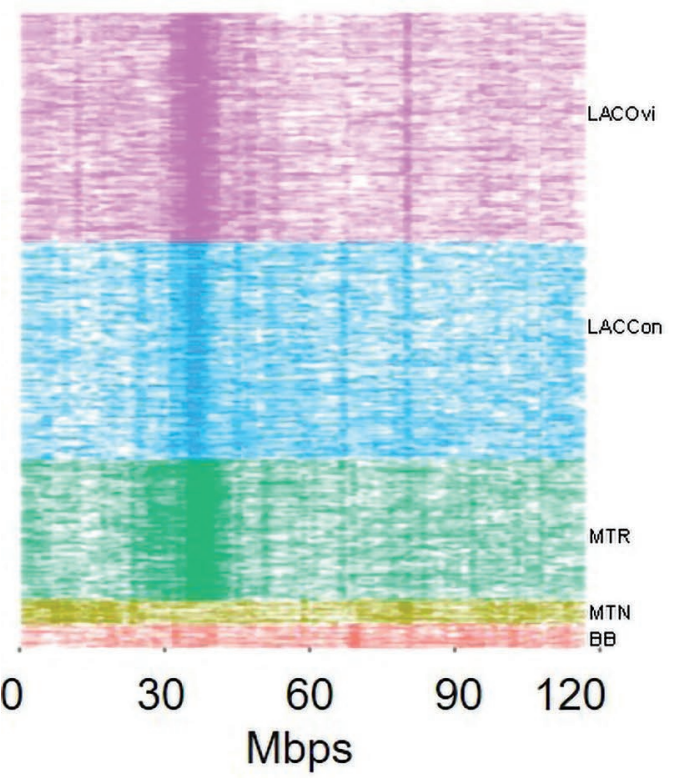

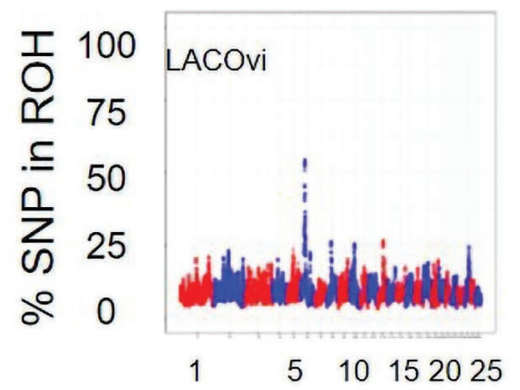
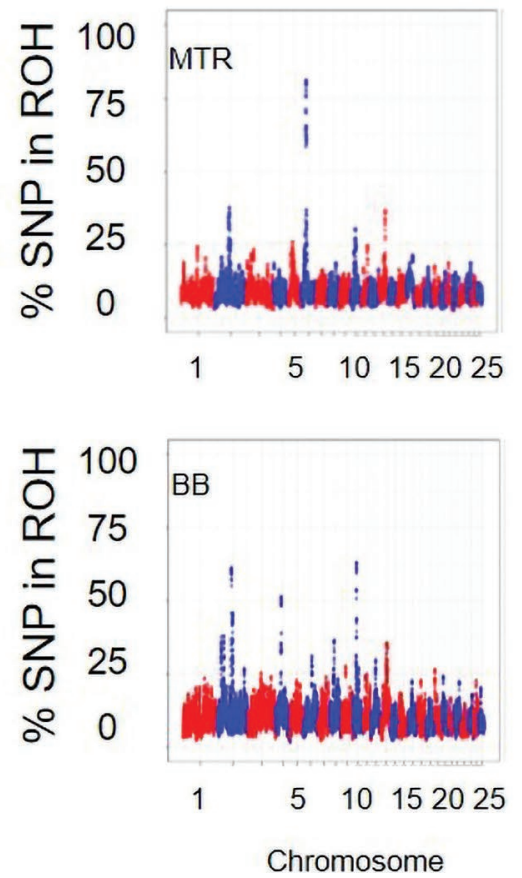
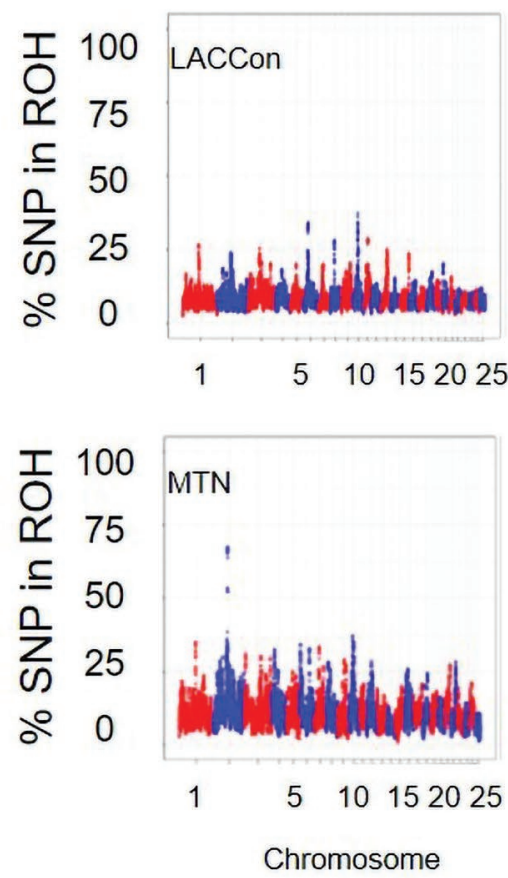

Chromosome

Figure 2. (a) Runs of homozygosity detected for each animal on OAR6. Mbps = megabases. (b) Percentage of times each SNP falls inside an ROH. BB = BascoBéarnaise; MTN = Manech Tête Noire; MTR = Manech Tête Rousse; LACCon = Lacaune Confederation; LACOvi = Lacaune Ovitest. 
shown in Table 1. The highest $\mathrm{F}_{\mathrm{ST}}$ values were observed between Western Pyrenees breeds and Lacaune subpopulations.

The allele frequencies observed for both SNP also diverged for the same allele between Western Pyrenees breeds (BB, MTN, and MTR) and Lacaune subpopulations (LACCon and LACOvi). For SNP OAR6_41583796.1, the allele frequency ranged between 0.62 and 0.95 in Western Pyrenees breeds and between 0.07 and 0.13 in Lacaune subpopulations for the same allele. In other words, they were nearly fixed for opposite alleles. Similar results were observed for SNP OAR6 41709987.1. In addition, both SNP were in almost complete linkage disequilibrium (calculated from allele frequencies, $\mathrm{R}^{2}=0.98$ ).

Figure $2 \mathrm{a}$ shows the $\mathrm{ROH}$ island detected for each animal on OAR6. The frequency of ROH was high between 30 and $40 \mathrm{Mb}$ in LACOvi and LACCon subpopulations and the MTR breed. This ROH island included both SNP OAR6 41583796.1 and OAR6 41709987.1 (the highest ranked SNP for $\overline{\mathrm{F}}_{\mathrm{ST}}$ ). The ROH island detected on OAR6 involves a large interval compared with the reduced interval obtained from the 2 most significant SNP in the $\mathrm{F}_{\mathrm{ST}}$ analysis. This suggests that the $\mathrm{F}_{\mathrm{ST}}$ approach is more precise than ROH methodology in terms of mapping accuracy. However, the gene mapping accuracy of $\mathrm{ROH}$ is affected by the criteria used to define an $\mathrm{ROH}$, which in turn depends on the available genotype SNP density. In any case, ROH signals suggest that the hitchhiking effect involves a large region of homozygosity around the potential candidate genes. Some agreement between $\mathrm{ROH}$ islands and selection signatures has been previously reported in the literature (e.g., Purfield et al., 2017). Other regions of high percentage of time each SNP falls inside an ROH were also detected in OAR2, OAR4, and OAR10 (Figure 2b), but no concordance between those homozygous regions and strong differentiation coefficient was observed. This lack of agreement could be attributed to demography and recombination events giving rise to ROH (Bosse et al., 2012).

The selection signal identified on OAR6 has been previously reported in the literature (e.g., Al-Mamun et al., 2015; Manunza et al., 2016; Gutiérrez-Gil et al., 2017). Fariello et al. (2014) found on OAR6 between 33.22 and $41.02 \mathrm{Mb}$ a signal of selection, and the candidate genes associated with this position were $A B C G 2$ (located in OAR6 between 36,514,210 and 36,556,824 bp on the OAR 3.1 reference genome), NCAPG (located in OAR6 between $37,256,548$ and $37,333,851$ bp on the OAR 3.1 reference genome), and $L C O R L$ (located in OAR6 between 37,365,236 and 37,452,332 bp on the OAR 3.1 reference genome). $A B C G 2$ has been associated with a strong QTL for milk production in cattle, and $N C A P G$ and $L C O R L$ have been associated with fetal growth (Eberlein et al., 2009) and calving ease in cattle (Druet et al., 2013). Plassais et al. (2019) detected a significant association with LCORL and height, weight, and life span in canids. Accordingly, NCAPG and LCORL genes have agricultural and adaptive importance and have been associated with allelic heterogeneity in selection signatures (Rochus et al., 2018).

Naval-Sanchez et al. (2018) analyzed 43 sheep breeds and 17 Asiatic mouflon with genome sequence resolution. On OAR6, between 37.42 and $37.51 \mathrm{Mb}$, they found a signature of selection, and the gene associated with this position was $L C O R L$, which has a function related to weight and height (as the $N C A P G$ gene). These authors indicated that domestic sheep contain a selective sweep in the $L C O R L$ promoter region, whereas mouflon contain a sweep downstream of $N C A P G$.

To conclude, $\mathrm{ROH}$ and single marker $\mathrm{F}_{\mathrm{ST}}$ analyses agreed that selection signatures exist around markers OAR6_41583796.1 and OAR6_41709987.1 that were located near the NCAPG and LCORL genes. One factor involved in shaping $\mathrm{ROH}$ patterns in French Dairy sheep was selection. Accordingly, the preliminary identification of ROH can suggest the presence of selection. However, for the identification of potential candidate genes, $\mathrm{ROH}$ detection should be combined with other approaches to improve mapping accuracy.

\section{References}

Al-Mamun, H. A., P. Kwan, S. A. Clark, M. H. Ferdosi, R. Tellam, and C. Gondro. 2015. Genome-wide association study of body weight in Australian Merino sheep reveals an orthologous region on OAR6 to human and bovine genomic regions affecting height and weight. Genet. Sel. Evol. 47:66. https://doi.org/10.1186/s12711-015-0142-4.

Biscarini, F., P. Cozzi, G. Gaspa, and G. Marras. 2018. Detect runs of homozygosity and runs of heterozygosity in diploid genomes. R package version 0.9.5. Accessed May 10, 2018. https://cran.r-project.org/web/packages/ detectRUNS/.

Bonhomme, M., C. Chevalet, B. Servin, S. Boitard, J. Abdallah, S. Blott, and M. SanCristobal. 2010. Detecting selection in population trees: The Lewontin and Krakauer test extended. Genetics 186:241-262. https://doi .org/10.1534/genetics.104.117275.

Bosse, M., H. J. Megens, O. Madsen, Y. Paudel, L. A. Frantz, L. B. Schook, R. P. Crooijmans, and M. A. Groenen. 2012. Regions of homozygosity in the porcine genome: Consequence of demography and the recombination landscape. PLoS Genet. 8:e1003100. https://doi.org/10.1371/journal.pgen .1003100 .

Chang, C. C., C. C. Chow, L. C. A. M. Tellier, S. Vattikuti, S. M. Purcell, and J. J. Lee. 2015. Second-generation PLINK: Rising to the challenge of larger and richer datasets. Gigascience 4:7. https://doi.org/10.1186/s13742-015 $-0047-8$.

Druet, T., L. Perez-Pardal, C. Charlier, and M. Gautier. 2013. Identification of large selective sweeps associated with major genes in cattle. Anim. Genet. 44:758-762. https://doi.org/10.1111/age.12073.

Eberlein, A., A. Takasuga, K. Setoguchi, R. Pfuhl, K. Flisikowski, R. Fries, N. Klopp, R. Fürbass, R. Weikard, and C. Kühn. 2009. Dissection of genetic factors modulating fetal growth in cattle indicates a substantial role of the non-SMC condensin I complex, subunit G (NCAPG) gene. Genetics 183:951-964. https://doi.org/10.1534/genetics.109.106476.

Fariello, M. I., B. Servin, G. Tosser-Klopp, R. Rupp, C. MorenoInternational Sheep Genomics Consortium, M. S. Cristobal, and S. Boitard. 2014. Selection signatures in worldwide sheep populations. PLoS One 9:e103813. https://doi.org/10.1371/journal.pone.0103813.

Gautier, M., and R. Vitalis. 2012. rehh: An R package to detect footprints of selection in genome-wide SNP data from haplotype structure. Bioinformatics 28:1176-1177. https://doi.org/10.1093/bioinformatics/bts115.

Gutiérrez-Gil, B., C. Esteban-Blanco, P. Wiener, P. K. Chitneedi, A. SuarezVega, and J. J. Arranz. 2017. High-resolution analysis of selection sweeps identified between fine-wool Merino and coarse-wool Churra sheep breeds Genet. Sel. Evol. 49:81. https://doi.org/10.1186/s12711-017-0354-x.

Howrigan, D. P., M. A. Simonson, and M. C. Keller. 2011. Detecting autozygosity through runs of homozygosity: A comparison of three autozygosity detection algorithms. BMC Genomics 12:460. https://doi.org/10.1186/ 1471-2164-12-460.

Legarra, A., G. Baloche, F. Barillet, J. M. Astruc, C. Soulas, X. Aguerre, F. Arrese, L. Mintegi, M. Lasarte, F. Maeztu, I. Beltrán de Heredia, and E. Ugarte. 2014. Within- and across-breed genomic predictions and genomic relationships for Western Pyrenees dairy sheep breeds Latxa, Manech, and Basco-Béarnaise. J. Dairy Sci. 97:3200-3212. https://doi.org/10.3168/jds .2013-7745

Lewontin, R. C., and J. Krakauer. 1973. Distribution of gene frequency as a test of the theory of the selective neutrality of polymorphisms. Genetics 74:175-195. https://doi.org/10.1093/genetics/74.1.175. 
Manunza, A., T. F. Cardoso, A. Noce, A. Martinez, A. Pons, L. A. Bermejo, V. Landi, A. Sanchez, J. Jordana, J. V. Delgado, S. Adan, J. Capote, O. Vidal, E. Ugarte, J. J. Arranz, J. H. Calvo, J. Casellas, and M. Amills. 2016. Population structure of eleven Spanish ovine breeds and detection of selective sweeps with BayeScan and hapFLK. Sci. Rep. 6:27296. https://doi.org/10 $.1038 /$ srep27296.

Nandolo, W., Y. T. Utsunomiya, G. Mészáros, M. Wurzinger, N. Khayadzadeh, R. B. P. Torrecilha, H. A. Mulindwa, T. N. Gondwe, P. Waldmann, M. Ferenčaković, J. F. Garcia, B. D. Rosen, D. Bickhart, C. P. van Tassell, I. Curik, and J. Sölkner. 2018. Misidentification of runs of homozygosity islands in cattle caused by interference with copy number variation or large intermarker distances. Genet. Sel. Evol. 50:43. https://doi.org/10.1186/ s12711-018-0414-x.

Naval-Sanchez, M., Q. Nguyen, S. McWilliam, L. R. Porto-Neto, R. Tellam, T. Vuocolo, A. Reverter, M. Perez-Enciso, R. Brauning, S. Clarke, A. McCulloch, W. Zamani, S. Naderi, H. R. Rezaei, F. Pompanon, P. Taberlet, K. C. Worley, R. A. Gibbs, D. M. Muzny, S. N. Jhangiani, N. Cockett, H. Daetwyler, and J. Kijas. 2018. Sheep genome functional annotation reveals proximal regulatory elements contributed to the evolution of modern breeds. Nat. Commun. 9:859. https://doi.org/10.1038/s41467-017-02809 -1 .

Plassais, J., J. Kim, B. W. Davis, D. M. Karyadi, A. N. Hogan, A. C. Harris, B. Decker, H. G. Parker, and E. A. Ostrander. 2019. Whole genome sequencing of canids reveals genomic regions under selection and variants influencing morphology. Nat. Commun. 10:1489. https://doi.org/10.1038/ s41467-019-09373-w.

Purfield, D. C., S. McParland, E. Wall, and D. Berry. 2017. The distribution of runs of homozygosity and selection signatures in six commercial meat sheep breeds. PLoS One 12:e176780. https://doi.org/10.1371/journal pone. 0176780 .

Rochus, C. M., F. Tortereau, F. Plisson-Petit, G. Restoux, C. Moreno-Romieux, G. Tosser-Klopp, and B. Servin. 2018. Revealing the selection history of adaptive loci using genome-wide scans for selection: An example from domestic sheep. BMC Genomics 19:71. https://doi.org/10.1186/s12864 $-018-4447-\mathrm{x}$.
Sabeti, P. C., D. E. Reich, J. M. Higgins, H. Z. Levine, D. J. Richter, S. F. Schaffner, S. B. Gabriel, J. V. Platko, N. J. Patterson, G. J. McDonald, H. C. Ackerman, S. J. Campbell, D. Altshuler, R. Cooper, D. Kwiatkowski, R. Ward, and E. S. Lander. 2002. Detecting recent positive selection in the human genome from haplotype structure. Nature 419:832-837. https://doi .org/10.1038/nature01140.

Voight, B. F., S. Kudaravalli, X. Wen, and J. K. Pritchard. 2006. A map of recent positive selection in the human genome. PLoS Biol. 4:e72. https://doi.org/ 10.1371/journal.pbio.0040072.

Weir, B. S., and C. C. Cockerham. 1984. Estimating F-statistics for the analysis of population structure. Evolution 38:1358-1370.

Zeder, M. A. 2008. Domestication and early agriculture in the Mediterranean basin: Origins, diffusion, and impact. Proc. Natl. Acad. Sci. USA 105:11597-11604. https://doi.org/10.1073/pnas.0801317105.

Zhang, Q., B. Guldbrandtsen, M. Bosse, M. S. Lund, and G. Sahana. 2015 Runs of homozygosity and distribution of functional variants in the cattle genome. BMC Genomics 16:542. https://doi.org/10.1186/s12864-015 $-1715-x$.

\section{Notes}

A. Legarra $\odot$ https://orcid.org/0000-0001-8893-7620

This study was supported by the European Unions' Horizon 2020 Research and Innovation program under grant agreement no. 772787-SMARTER, ARDI (Ardi es Research, Development, Innovation; grant agreement EFA 208/16) from POCTEFA (Programa Operativo de Cooperación Territorial España Francia Andorra) funds, and GDivSelGen action (INRA SelGen metaprogram).

We thank 2 anonymous reviewers for useful suggestions on the manuscript. The GenoToul bioinformatics platform Toulouse Midi-Pyrenees provided computing and storage resources.

The authors declare no conflicts of interest. 